\title{
Determinants Of Low Apgar Score In Newborns Delivered At Lemlem Karl General Hospital, Northern Ethiopia, 2018: A Case Control Study
}

Mussie Mulugeta Gebremedhin ( $\nabla$ mussiemulugeta@gmail.com )

Axum University

Mengistu Welday Gebremichael

Mekelle University

Berhane Gebreegizabiher Gebremichael

Mekelle University College of Health Sciences

Mihrete-ab Mehari Reda

Mekelle University College of Health Sciences

Tesfay Adhena Hailu

Aksum University

Gebrekiros Aregawi Gebremeskel

Aksum University

Kenean Getaneh Tilaye

Woldia University

Henok Kumsa Meikena

Woldia University

Nigus Bililign Yimer

Woldia University

Research note

Keywords: Determinant, Low Apgar score, Ethiopia

Posted Date: November 14th, 2019

DOI: https://doi.org/10.21203/rs.2.17207/v1

License: (c) (i) This work is licensed under a Creative Commons Attribution 4.0 International License. Read Full License 


\section{Abstract}

OBJECTIVE: This study is aimed to investigate determinant factors of low Apgar score in newborns delivered at Lemlem Karl General Hospital, Tigray National Regional State, Ethiopia; 2018. RESULTS: Socio-demographic characteristics of mothers were not significantly associated. However, antepartum hemorrhage (AOR=3.509; $95 \% \mathrm{Cl} 1.526-8.067$ ), pregnancy induced hypertensive disorders (AOR=2.69; 95\% $\mathrm{Cl} 1.351-5.357$ ), prolonged second stage of labor (AOR=2.630; 95\% Cl 1.399-4.944), meconium stained liquor (AOR=6.955; 95\% $\mathrm{Cl} 3.721$ 13.001)) \& low birth weight (AOR=4.380; $95 \% \mathrm{Cl} 2.216-8.657)$ were significantly associated with low Apgar score. Some obstetric factors \& one fetal factor were significantly associated with low Apgar score. Therefore, improving labor management of mothers those who are diagnosed with antepartum hemorrhage, pregnancy induced hypertensive disorders, prolonged second stage of labor, and meconium stained liquor is recommended. KEY WORDS : Determinant, Low Apgar score, Ethiopia.

\section{Introduction}

The first minutes after birth are crucial for newborns' adaptation to extra-uterine life. Therefore during this period, reliable and objective tools are required to assess its clinical state. Dr. Virginia Apgar created the Apgar scoring system in 1952 as a method of evaluation of newborns (1). The Apgar score (AS) comprises five components Color, Heart Rate, Reflexes, Muscle Tone, Respiration; each assigned a score of 0, 1, or 2 (2). AS ranging from 710 are considered healthy. The score is reported at $1^{\text {st }}$ and $5^{\text {th }}$ minutes after birth for all infants, and at 5 minute interval thereafter until 20 minutes for infants with a score of less than 7(2).

Low AS indicates abnormal conditions in newborns but do not suggest a specific etiology. Low $1^{\text {st }}$ minute AS has not been found to correlate with future adverse outcomes. However, low $5^{\text {th }}$ minute AS is associated with increased mortality in premature newborns (4-8), and also associated with various types of future developmental and cognitive problems (9).

Since it is the only form of evaluation in developing countries, where laboratory tests may not be available, the low cost of the AS is useful in identifying children who need additional care, even in the absence of laboratory data (10).

However, despite its undeniable usefulness, AS can be influenced by anesthesia, congenital malformation, gestational age, trauma, and inter-observer variability (3). Elements of the score such as tone, color, and reflex irritability can be subjective, and partially depend on the physiologic maturity of the newborn (2).

\section{Methods}

\section{Study design and settings}

An institutional based unmatched case control study was conducted on 221 cases and 441 controls, with total sample size of 662 from September 2017 to January 2018. Lemlem Karl general hospital is found in Maychew town, located $662 \mathrm{~km}$ north of Addis Ababa the capital of Ethiopia.

\section{Sample size and sampling procedures}


All neonates born after 34 completed weeks of gestation in Lemlem Karl hospital were included in the study. However, newborns with gross congenital anomalies incompatible with life, neonates born via elective cesarean section (CS), deliveries of unknown gestational age (unknown last normal menstrual period and no ultrasound estimation), twins, neonates born to mothers with severe medical diseases during the pregnancy period and newborns, who had not been given an AS at fifth minutes of birth were excluded from the study. The required sample size was calculated using the double population proportion formula by using Open Epi statistical software by considering $80 \%$ power, $95 \%$ confidence level, ratio of controls to cases $2: 1$, the proportion of cases born to grand multiparous mothers $12.09 \%$ and proportion of controls born to grand multiparous mothers $5.4 \%$ taken from a previous study in Tanzania (31), and with odds ratio of 2.41; the final sample size was 662 newborns (221 cases \& 441 controls). First The medical record numbers (MRN) of the mothers of both cases' and control' were identified from birth registration book of maternity unit of the hospital those registered from September 11, 2014 to September 10, 2017. The fifth minute AS recorded was used as a measure of infant's health status at birth. Accordingly 679 charts of the mothers of cases' and 4,004 charts of the mothers of controls' were identified. Then systematic sampling method was employed to select cases and controls by considering skip intervals of 3 and 9 respectively. If the medical chart is missed or not eligible, immediate neighbor medical chart that host eligible newborn was selected.

\section{Measurement}

\section{Low Apgar score: a score less than seven at fifth minute of birth (1).}

\section{Birth weight: was classified as low birth weight (LBW) (< 2500 gram), normal birth weight (2500 - 3999 gram) and macrosomia (>4000 gram) (43).}

Prolonged second stage of labour: it was defined as no progress (descent or rotation) after full cervical dilatation women for $\geq 4$ hours with and $\geq 3$ hours without epidural anesthesia in nulliparous and for $\geq 3$ hours with and $\geq 2$ hours without epidural anesthesia in multiparous mothers (43).

\section{Data collection tools and techniques}

Data was collected through pretested and structured checklist. A total of 8 data collectors (BSc. in Midwifery) were involved in the data collection process. Furthermore, three MSc holder midwives were recruited as supervisors. The purposes and objectives of the study were clearly explained to the hospital managers and staffs.

\section{Data quality controls}

The checklist was pretested on 34 medical charts in Alamata general hospital out of the study area. Eight data collectors and three supervisors were selected from Lemlem Karl general hospital and received a total of 5 days of intensive training before data collection. During data collection, any personal identifiers were not recorded. 


\section{Data processing and analysis}

After the data collection, data was entered in to Epi Info version 3.5.1 and exported to SPSS version 22.0 for analysis. Both bivariable and multivariable logistic regression analysis were carried out to identify determinant factors of low Apgar score. In bivariate logistic regression analysis, variables with p-value less than $20 \%$ were considered into the multivariable analysis to control the possible effect of confounders. Adjusted odds ratio (AOR) with a $95 \%$ confidence interval $(\mathrm{Cl})$ was calculated to see the strength and significant association. Variables having a p-value less than 0.05 in the multivariable logistic regression analysis were considered as statistically significant.

\section{Results}

\section{Socio-demographic characteristics of the study Participants}

A total of 662 cards of newborns' mothers (221 cases and 441 controls) were included in this study. The mean $(+S D)$ age of mothers was $28(+6)$ and $27(+5)$ years among cases and controls respectively. Moreover the age of mothers of cases and controls was in the range of (16-45 years) and (16-47 years) respectively (Table S1).

\section{Obstetric characteristics of mother's among cases and controls}

The percentage of mothers among cases with prolonged second stage of labor (PSSL) 35(15.8\%) was lower than the percentage of the mothers of controls $27(6.1 \%)$. Additionally, $7.2 \%$ of mothers among cases and $3.2 \%$ of mothers among controls were diagnosed with APH in the current pregnancy. More than one-fifth $(21.7 \%)$ mothers in cases and 25(5.7\%) mothers in controls were diagnosed with PIHD (Table 1).

\section{Characteristics of newborns}

Of all study subjects, $48.4 \%$ of cases and more than half of controls were females. Furthermore, regarding the gestational age (GA) three-fourth (78.3\%) of cases and majority (89.8) of controls were term (Table 2 ).

\section{Determinant factors for low Apgar score in newborns}

Both bivariable and multivariable logistic regression analyses were done to see the association of the selected variables and low AS. As it is shown in Table 3, age of mothers, Parity, APH, PIHD, PROM, onset of labor, PSSL, MSL, mode of delivery, GA, fetal presentation, and fetal birth weight had significant association with low AS in the bivariable analysis. However, in the multivariable logistic regression analysis APH, PIHD, PSSL, MSL, mode of delivery, and fetal birth weight were significantly and independently associated with low AS. Accordingly, the odds of developing low AS among neonates born to mothers with PSSL were 2.63 times (AOR $=2.63 ; 95 \% \mathrm{Cl}$ 1.399-4.944) higher compared to their counterparts. Moreover, the likelihood of encountering low AS was 3.5 times (AOR $=3.509 ; 95 \% \mathrm{Cl} 1.526-8.067)$ higher among neonates born to mothers those who diagnosed with $\mathrm{APH}$. 
In this study, the higher odds of developing low AS were also observed among neonates born to mothers with PIHD (AOR = 2.69; 95\% CI 1.351-5.357) than newborns born to mothers without PIHD. Likewise, the odds of exhibited low AS among neonates with low BW were 4.38 times (AOR = 4.38; 95\% CI 2.216-8.657)higher compared to those of their counterparts (Table 3).

\section{Discussion}

This study revealed that neonates born to mothers those who diagnosed with APH were more than three times at higher odds for low AS. This finding is in agreement with findings of the case control studies done in Nigeria and India which showed statistically significant association between APH and low AS $(39,40)$. Nevertheless, APH was not significantly associated with low AS in one Brazilian study(15). This controversy might be due to the difference in sample sizes, socio-economic factors and the good health system and interventions to decrease neonatal morbidity in the developed world.

Moreover, PIHD increased the odds of low AS by two-folds. This finding is in line with the studies done in Sweden and India; which showed statistically significant association between PIHD and low AS $(29,38)$.

Furthermore, neonates born to mothers with PSSL were more than two times more likely to have low AS than neonates born to mothers without PSSL. Similar findings were reported in studies from Brazil, Sweden and USA $(15,19,34,35)$. In the retrospective cohort and case-control study done in Brazil, PSSL increased low AS by three folds (15). Whereas in the Swedish population-based cohort study, newborns born to mothers with PSSL were 2 times more likely to have low AS (19). This might be due to the fact that in prolonged second stage of labor, there will be chance of caesarean or manipulative vaginal deliveries and a chance of fetal distress, which could further affect AS.

This study also revealed that MSL during labor increased low AS by seven folds. Similar finding was reported from a cohort study in Australia, which implies, newborns born to mothers with MSL during labor were 3 times more likely to have low AS (30). Another study done in Brazil found no association between low AS and MSL (15). This controversy might be due to the fact that the Brazilian finding was reported from relatively small sample size. Moreover, it could be also due to the good health system and interventions to decrease neonatal morbidity in the developed world.

Newborns born via cesarean delivery (CS) were about 2 times at higher odds for low AS. This finding is consistent with findings of studies from Brazil and Sweden $(36,37)$. According to the finding of the Brazilian study, CS increased probability of low AS by 3 folds (36). In another study, CS increased low AS by seven folds (37). The difference in magnitude between this and the Swedish study may be attributed to the fact that the Swedish study was conducted on very large sample size than this study. The association of cesarean delivery and low AS may be due to the lack of fetal chest compression during cesarean delivery which facilitates lung clearance from secretions and fluids. Similarly, drugs used for anesthesia during CS can also decrease uterine and placental circulation then it causes fetal hypoxemia which further leads to fetal morbidity including low AS (43).

Moreover, this study also revealed newborns born with LBW were 4 times more likely to have low AS. This finding is similar with a study done in North West Ethiopia, which showed newborns born with LBW were 4 times more likely to have low AS (21). This could be explained by the fact that small babies might suffer from difficult 
birthing and might develop difficulty in cardiopulmonary transition and perinatal asphyxia which predispose the newborns to various complications including low AS (43).

\title{
Conclusion
}

APH, PIHD, PSSL, MSL and LBW were significantly associated with low AS. Therefore, improving obstetrical care service of mothers those who are diagnosed with the significantly associated cases is recommended. Health professional have to keep their strength on early detection and management of newborns with low AS to prevent further complications.

\section{Limitation}

As a retrospective design, the study was limited to the available data in the delivery register. So, this study did not consider some potential risk factors for low AS. Moreover, there are additional limitations including difficulty of generalizing to births that did not happen at the hospital, lack of control for all aspects of socio-economic factor and access to care, error in medical records that misclassify cases/controls.

\section{Abbreviations}

\section{AOR: Adjusted Odds Ratio}

\section{APH: Antepartum Hemorrhage}

\section{AS: Apgar score}

BSc: Bachelor's Degree in Science

\author{
CHF: Congestive Heart Failure
}

\section{Cl: Confidence Interval}

\section{COR: Crude odds Ratio}

\section{CS: Cesarean Section}

\section{DM: Diabetic Mellitus}

\section{EDHS: Ethiopian Demographic and Health Survey}


ETT: Endotracheal tube

FMOH: Federal Ministry of Health

IUGR: Intra uterine retardation

UN IGME: United Nations Inter-agency Group for Child Mortality Estimation

USA: United States of America

MRN: Medical Record Number

MSc: Master's Degree in Science

MSL: Meconium Stained Liquor

NCPAP: Nasal continuous positive airway pressure

NGO: Non-Governmental Organization

NICU: Neonatal Intensive Care Unit

OR: Odds Ratio

PIHD: Pregnancy Induced Hypertensive Disorders

PPROM: Preterm Premature Rupture of Membrane

PPV: Positive-pressure ventilation

PROM: Premature Rupture of Membrane

PSSL: Prolonged Second Stage of labor 


\section{WHO: World Health Organization}

\section{Declarations}

\section{Ethics approval and consent to participate}

Ethical approval was obtained from Institutional Research Review Board of Mekelle University College of health science. Data was collected after permission was gained from the medical director of the hospital. Moreover confidentiality and anonymity were assured by analyzing and disseminating the data in aggregate. Since the study was retrospective by design, written informed consent was not obtained from the patient.

\section{Consent for publication}

\section{Not applicable, no individual detail is presented.}

\section{Availability of data and material}

All pertinent data was in the manuscript. The datasets investigated during this study was available from the corresponding author Mussie Mulugeta: mussiemulugeta@gmail.com.

\section{Competing interests}

\section{No opposing interests}

\section{Funding}

The study was funded by Mekelle University that was not involved in the design of the study, data collection, analysis and interpretation.

\section{Authors' contributions}

MMG: Developed the proposal, analyzed data, and wrote the report and the manuscript. MWG and BGG: Organized overall process. TAH, GAG, M-AMR, KGT, NBY and HKM: Contributed in proposal writing, data collection and analysis. All authors checked and accepted the final manuscript.

\section{Acknowledgements}

We would like to thank Mekelle University for facilitating conditions and financial support to prepare this study. We would also like to pass our gratitude towards all staffs of Tigray regional state health bureau, Lemlem Karl General Hospital, data collectors, and supervisors who gave us their unduly support, critical comments and helpful information. 


\section{Authors' information}

MMG: MSc in Clinical Midwifery, Department of Midwifery, College of Health Sciences, Aksum University, Aksum, Ethiopia. Email: mussiemulugeta@gmail.com

MWG: Phd in public health, Department of Midwifery, College of Health Sciences, Mekelle University, Mekelle, Ethiopia. Email: mengsteabw@gmail.com

BGG: MSc in Clinical Midwifery, Department of Midwifery, College of Health Sciences, Mekelle University, Mekelle, Ethiopia. Email: naodmgb@gmail.com

TAH: MSc in Clinical Midwifery, Department of Midwifery, College of Health Sciences, Aksum University, Aksum, Ethiopia. Email: tessotesa@gmail.com

GAG: MSc in Maternity and Neonatal Nursing, Department of Midwifery, College of Health Sciences, Aksum University, Aksum, Ethiopia. Email: gkaregawi@gmail.com

M-AMR: MSc in Clinical Midwifery, Department of Midwifery, College of Health Sciences, Mekelle University, Mekelle, Ethiopia. Email: meharimerry24@gmail.com

KGT: MSc in Maternity Nursing, Department of Nursing, Faculty of Health Sciences, Woldia University, Woldia, Ethiopia. Email: keneanget@gmail.com

HKM: MSc in Clinical Midwifery, Department of Midwifery, Faculty of Health Sciences, Woldia University, Woldia, Ethiopia. Email: henokkumsa@gmail.com

NBY: MSc in Clinical Midwifery, Department of Midwifery, Faculty of Health Sciences, Woldia University, Woldia, Ethiopia. Email: bililignnigus@gmail.com

\section{References}

1. Apgar V. A proposal for a new method of evaluation of the newborn infant. Currresanesthanalg. 1953;32(4):260-7.

2. American Academy of Pediatrics and American Heart Association. Textbook of Neonatal Resuscitation. 6th editio. ELK Grove Village II, editor. American Academy of Pediatrics and American Heart Association; 2011.

3. American College of obstetrics and Gynecology, Task Force on Neonatal Encephalopathy, American Academy of Pediatrics. Neonatal Encephalopathy and Neurologic Outcome. 2nd editio. Washington DC: American College of obstetricians and Gynecologists; 2014.

4. Oliveira TG de, Freire PV, Moreira FT, Moraes J da SB de, Arrelaro RC, Rossi S, et al. Apgar score and neonatal mortality in a hospital located in the southern area of São Paulo city, Brazil. Einstein (São Paulo) [Internet]. 2012;10(1):22-8. Available from: http://www.scielo.br/scielo.php?Script = sci_arttext\&pid = S167945082012000100006\&lng = en\&tlng = en

5. Ehrenstein V. Association of Apgar scores with death and neurologic disability. Clin Epidemiol [Internet]. 2009;1:45-53. Available from: 
http://www.ncbi.nlm.nih.gov/pubmed/20865086\%5Cnhttp://www.pubmedcentral.nih.gov/articlerender.fcgi? Artid $=$ PMC2943160

6. Fernández-carrocera LA, Guevara-fuentes CA, Salinas-ramírez V. Risk factors associated with mortality in infants weighing less than $1500 \mathrm{~g}$ using the CRIB II scale. Bol Med Hosp Infant Mex. 2011;68(5):330-6.

7. Abdullah A, Hort K, Butu Y, Simpson L. Risk factors associated with neonatal deaths: a matched case control study in Indonesia. Glob Heal Action. 2016;1(3):1-12.

8. Maria P, Soares S, Maestá I, Maria L, Suppo S, Felipe L, et al. Risk factors for perinatal death in two different levels of care: a case-control study. Reprod Health. 2014;11(11):1-7.

9. Suokas FE. The effects of Apgar score defined asphyxia on adulthood cognition: A longitudinal study. Ethesis (opinnäytteet), ethesis.helsinki.fi. 2017;1:23-26.

10. Cunha AA, Fernandes DS, Melo PF GM. Fatores associados à asfixia perinatal. Rev Bras Ginecol Obs. 2004;26(10):799-805.

11. American College of Obstetrics and Gynecology, American Academy of Pediatrics. The Apgar Score. In American College of obstetricians and Gynecologists and American Academy of Pediatrics; 2015. P. 1-4.

12. Estimates Developed by the UN Inter-agency Group for Child Mortality Estimation. Levels and Trends in Child Mortality. New York city; 2015.

13. Central Statistical Agency, The DHS Program ICF. Ethiopian Demographic and Health Survey 2016. Addis Ababa, Ethiopia; 2017.

14. Costa TL, Mota A, Duarte S, Araujo M, Ramos P, Machado HS, et al. Predictive Factors of Apgar Scores below 7 in Newborns: Can We Change the Route of Current Events? J Anesth Clin Res. 2016;7(10).

15. SALUSTIANO EMA, CAMPOS JADB, SILVIA MARIA IBIDI RR, ZUGAIB M. Low Apgar scores at 5 minutes in a low risk population: maternal and. Rev Assoc Med Bras. 2012;58(5):587-93.

16. Ehrenstein V, Pedersen L, Grijota M, Nielsen GL, Rothman KJ, Sørensen HT. Association of Apgar score at five minutes with long-term neurologic disability and cognitive function in a prevalence study of Danish conscripts. BMC Pregnancy Childbirth. 2009;9(14):1-7.

17. Bakhsha F, Yousefi Z, Aryaie M, Jafari SY. Comparison of Apgar score in new born by vaginal delivery and spinal anesthesia and its relationship with contributing factors. J Bas Res Med Sci. 2016;3(1):10-5.

18. Susilo SA, Pratiwi KN, Fattah ANA, Irwinda R, Wibowo N. Determinants of low APGAR score among preeclamptic deliveries in Cipto Mangunkusumo Hospital : a retrospective cohort study in 2014. Med J Indones. 2015;24(3):183-9.

19. Altman M. Prolonged second stage of labor is associated with low Apgar score Corresponding author. Eur $\mathrm{J}$ Epidemiol. 2015;1-19.

20. Dassah ET, Odoi AT, Opoku BK. Stillbirths and very low Apgar scores among vaginal births in a tertiary hospital in Ghana: a retrospective cross-sectional analysis. BMC Pregnancy Childbirth. 2014;14(289):1-7.

21. Gudayu TW. Proportion and factors associated with low fifth minute Apgar score among singleton newborn babies in Gondar University referral hospital; North West Ethiopia. Afr Health Sci. 2017;17(1):6-11.

22. Kotlicka-antczak M, Smigielski J. Obstetrical complications and Apgar score in subjects at risk of psychosis. J Psychiatr Res. 2014;48:79-85.

23. Li J, Cnattingus S, Gissler M, Vestergaard M, Obel C, Ahrensberg J, et al. The 5-minute Apgar score as a predictor of childhood cancer: a population-based cohort study in fi ve million children. BMJ Open. 
2012;0:1-8.

24. Eun S, Lee JM, Yi DY, Lee NM, Kim H, Yun SW, et al. Assessment of the association between Apgar scores and seizures in infants less than 1 year old. Seizure Eur J Epilepsy [Internet]. BEA Trading Ltd; 2016;37:4854. Available from: http://dx.doi.org/10.1016/j.seizure.2016.03.001

25. Zarkesh $\mathrm{M}$, Momtazbakhsh $\mathrm{M}$, Mojtabai $\mathrm{H}$. Incidence and risk factors of pneumothorax in premature low birth weight infants under mechanical ventilation. Iran J Neonatol. 2013;4(3):1-6.

26. Pan C, Qian D, Zhu H, Yu J, Liu H. Apgar score and reduced vision in children aged 3 to 6 years. Graefes Arch Clin Exp Ophthalmol. Graefe's Archive for Clinical and Experimental Ophthalmology; 2017;255(2):401-5.

27. SIAKWA M, KPIKPITSE, D. M. NEONATAL SEPSIS IN RURAL GHANA: A CASE CONTROL STUDY OF RISK FACTORS IN A BIRTH COHORT. Int J Res Med Heal Sci. 2014;4(5):77-88.

28. Straube S, Voigt M, Jorch G, Hallier E, Briese V, Borchardt U. Investigation of the association of Apgar score with maternal socio-economic and biological factors: an analysis of German perinatal statistics. Arch Gynecol Obs. 2010;282:135-41.

29. Svenvik M, Brudin L, Blomberg M. Preterm Birth: A Prominent Risk Factor for Low Apgar Scores. Biomed Res Int. 2015;2015:8.

30. Lai S, Flatley C, Kumar S. Perinatal risk factors for low and moderate five - minute Apgar scores at term. J.ejogrb. 2017;1(8).

31. Mgaya AH, Massawe SN, Kidanto HL, Mgaya HN. Grand multiparity: is it still a risk in pregnancy? BMC Pregnancy Childbirth. 2013;13(241):1-8.

32. Aragaw YA, Mahtemsilllasie M, Jarso H. Gynecology \& Obstetrics Grand Multiparity and Pregnancy Related Complications among Women Who Gave Birth at Jimma University Specialized Hospital, Jimma,. Gynecol Obs. 2017;7(4):1-6.

33. Rahmanian V, Ghasvari M, Abari P. ASSOCIATION OF APGAR SCORE WITH DELIVERY MODE IN THE NON DISTRESS NEWBORNS. Online J Biol Sci. 2014;14(1):21-5.

34. Cheng YW, Hopkins LM, Jr RKL, Caughey AB. Duration of the second stage of labor in multiparous women: maternal and neonatal outcomes. Am J Obstet Gynecol ogy. 2014;196:1-6.

35. Frisell T, Cnattingius S, Stephansson O. Prolonged second stage of labor is associated with low Apgar score. Eur J Epidemiol. 2015;1209-15.

36. ZORZ PDM DE, MAD JM, ROMBALDI RL. Perinatal factors associated with ph<7.1 in umbilical artery and Apgar 5 min < 7.0 in term newborn Artigo. Rev Bras Ginecol Obs. 2012;34(8):381-5.

37. Berglund S, Pettersson H, Cnattingius S, Grunewald C. How often is a low Apgar score the result of substandard care during labour? BJOG. 2010;117:968-78.

38. Vats K, Paul M. Study of fetal outcome in hypertensive disorders of pregnancy in a tertiary care maternity hospital of Delhi. Int J Reprod Contraception, Obstet Gynecol. 2016;5(11):3773-7.

39. Adekanle DA, Adeyemi AS, Fadero FF. Ante-partum haemorrhage and pregnancy outcome in LAUTECH Teaching Hospital, southwestern Nigeria. J Med Med Sci. 2011;2(December):1243-7.

40. Maiti S, Kanrar P, Karmakar C, Bagdi S. Maternal and Perinatal Outcome in Rural Indian Women with Placenta Previa. Br Biomed Bull. 2014;2(4):714-8.

41. Akintayo AA, Awoleke JO, Ogundare EO, Olatunya OS, Aduloju OP. PRETERM BIRTHS IN A RESOURCE CONSTRAINED SETTING : SOCI- OBIOLOGIC RISK FACTORS AND PERINATAL OUTCOMES. GHANA Med J. 
2015;49(4).

42. Human resource department. Lemlem Karl General Hospital Profile. 2017.

43. Uptodate21.2.

\section{Tables}

RESULTS

Table 1: Obstetric characteristics of mothers' among cases and controls at Lemlem Karl General Hospital, Maychew Town, Tigray, Etiopia; from September 11, 2014 - September 10, 2017. (N=662 (Cases=221, Controls= 441))

\begin{tabular}{|c|c|c|c|c|}
\hline \multirow[b]{2}{*}{ iables } & \multirow[b]{2}{*}{ Categories } & \multicolumn{2}{|c|}{ Fifth Minute Apgar Score } & \multirow[b]{2}{*}{$\begin{array}{l}\text { Total } \\
\text { N (\%) }\end{array}$} \\
\hline & & $\begin{array}{l}\text { Cases } \\
\text { N (\%) }\end{array}$ & $\begin{array}{c}\text { Controls } \\
\text { N (\%) }\end{array}$ & \\
\hline \multirow[t]{3}{*}{ ity } & Primipara & $102(46.2)$ & $188(42.6)$ & $290(43.8)$ \\
\hline & Multipara & $96(43.4)$ & $220(49.9)$ & $316(47.7)$ \\
\hline & Grandmultipara & $23(10.4)$ & $33(7.5)$ & $56(8.5)$ \\
\hline \multirow[t]{2}{*}{$\mathrm{H}$} & Yes & $16(7.2)$ & $14(3.2)$ & $30(4.5)$ \\
\hline & No & $205(92.8)$ & $427(96.8)$ & $632(95.5)$ \\
\hline \multirow[t]{2}{*}{ gnancy induced hypertensive disorder } & Yes & $48(21.7)$ & $25(5.7)$ & $73(11.0)$ \\
\hline & No & 173(78.3) & $416(94.3)$ & $589(89.0)$ \\
\hline \multirow{2}{*}{ mature Rupture of membrane (PROM) } & Yes & $28(12.7)$ & $30(6.8)$ & $58(8.8)$ \\
\hline & No & 193(87.3) & $411(93.2)$ & $604(91.2)$ \\
\hline \multirow[t]{2}{*}{ ation of the PPROM } & $<12$ hours & $11(39.3)$ & $12(40.0)$ & $23(39.7)$ \\
\hline & $>=12$ hours & 17(60.7) & $18(60.0)$ & $35(60.3)$ \\
\hline \multirow[t]{2}{*}{ set of labor } & Spontaneous & $184(83.3)$ & $412(93.4)$ & $596(90.0)$ \\
\hline & Induced & $37(16.7)$ & $29(6.6)$ & $66(10.0)$ \\
\hline \multirow[t]{2}{*}{ longed second stage of labor } & Yes & $35(15.8)$ & $27(6.1)$ & $62(9.4)$ \\
\hline & No & $186(84.2)$ & 414(93.9) & $600(90.6)$ \\
\hline \multirow[t]{2}{*}{ conium stained liquor } & Yes & $50(22.6)$ & $18(4.1)$ & $68(10.3)$ \\
\hline & No & 171(77.4) & 423(95.9) & $594(89.7)$ \\
\hline \multirow[t]{2}{*}{ jmentation of labor } & Yes & $25(11.3)$ & $44(10.0)$ & $69(10.4)$ \\
\hline & No & 196(88.7) & $397(90.0)$ & 593(89.6) \\
\hline \multirow[t]{4}{*}{ de of delivery } & Spontaneous Vaginal Delivery & $99(44.8)$ & $313(71.0)$ & $412(62.2)$ \\
\hline & Operative Vaginal Delivery & $39(17.6)$ & $47(10.7)$ & $86(13.0)$ \\
\hline & $\begin{array}{l}\text { Caesarean Delivery } \\
\text { Vaginal Breech Delivery }\end{array}$ & $69(31.2)$ & $73(16.6)$ & $142(21.5)$ \\
\hline & & $14(6.3)$ & $8(1.8)$ & $22(3.3)$ \\
\hline
\end{tabular}

Table 2: Characteristics of Newborns Who Delivered At Lemlem Karl General Hospital, Mychew Town, Tigray, Ethiopia; September, 2014 - September, 2017. $(N=662($ Cases $=221$, Controls= 441) $)$ 


\begin{tabular}{|c|c|c|c|c|}
\hline \multirow[b]{2}{*}{ Variables } & \multirow[b]{2}{*}{ Categories } & \multicolumn{2}{|c|}{ Fifth Minute Apgar Score } & \multirow[b]{2}{*}{$\begin{array}{l}\text { Total } \\
\text { N (\%) }\end{array}$} \\
\hline & & $\begin{array}{l}\text { Cases } \\
\text { N (\%) }\end{array}$ & $\begin{array}{c}\text { Controls } \\
\text { N (\%) }\end{array}$ & \\
\hline \multirow[t]{2}{*}{ Sex } & Female & $107(48.4)$ & $228(51.7)$ & $335(50.6)$ \\
\hline & Male & $114(51.6)$ & $213(48.3)$ & $327(49.4)$ \\
\hline \multirow[t]{3}{*}{ Gestational Age } & Late Preterm (34-36+6 weeks) & $41(18.6)$ & $29(6.6)$ & $70(10.6)$ \\
\hline & Term & 173(78.3) & 396(89.8) & $569(86.0)$ \\
\hline & Post term & $7(3.2)$ & 16(3.6) & $23(3.5)$ \\
\hline \multirow[t]{3}{*}{ Fetal presentation } & Breech & $23(10.4)$ & $11(2.5)$ & $34(5.1)$ \\
\hline & Vertex & 196(88.7) & $427(96.8)$ & $623(94.1)$ \\
\hline & Non-Vertex* & $2(0.9)$ & $3(0.7)$ & $5(0.8)$ \\
\hline \multirow[t]{3}{*}{ Birth weight } & $<2500$ gram & $52(23.5)$ & $24(5.4)$ & $76(11.5)$ \\
\hline & 2500 - 3999 gram & $166(75.1)$ & 413(93.7) & $579(87.5)$ \\
\hline & $\geq 4000$ gram) & $3(1.4)$ & $4(0.9)$ & $7(1.1)$ \\
\hline
\end{tabular}

*= Brow presentation, Shoulder presentation

Table 3: Bivariate and multivariate analysis of determinant factors for low Apgar score in newborns delivered at Lemlem Karl General Hospital, Maychew Town, Tigray, Ethiopia, 2018. 


\begin{tabular}{|c|c|c|c|c|}
\hline \multirow[t]{2}{*}{ Variables } & \multirow[t]{2}{*}{$\begin{array}{l}\text { Cases } \\
\text { no(\%) }\end{array}$} & \multirow[t]{2}{*}{ Controls no(\%) } & \multicolumn{2}{|c|}{ Odds ratios $(95 \% \mathrm{CI})$} \\
\hline & & & Unadjusted & Adjusted \\
\hline \multicolumn{5}{|l|}{ Age of mothers(Yrs.) } \\
\hline $20-34$ & $188(85.1)$ & $385(87.3)$ & Ref & Ref \\
\hline$<=19$ & $10(4.5)$ & $25(5.7)$ & $0.819(0.385-1.741)$ & $0.711(0.279-1.865)$ \\
\hline$\geq 35$ & $23(10.4)$ & $31(7.0)$ & $1.519(0.862-2.678)$ & $1.646(0.728-3.724)$ \\
\hline \multicolumn{5}{|l|}{ Parity } \\
\hline Multipara & $96(43.4)$ & $220(49.9)$ & Ref & Ref \\
\hline Primipara & $102(46.2)$ & $188(42.6)$ & $1.243(0.885-1.747)$ & $1.120(0.723-1.734)$ \\
\hline Grandmultipara & $23(10.4)$ & $33(7.5)$ & $1.597(0.891-2.864)$ & $1.595(0.723-1.734)$ \\
\hline \multicolumn{5}{|l|}{ Antepartum hemorrhage } \\
\hline Yes & $16(7.2)$ & $14(3.2)$ & $2.380(1.140-4.971)$ & $3.509(1.526-8.067) *$ \\
\hline No & 205(92.8) & $427(96.8)$ & Ref & Ref \\
\hline \multicolumn{5}{|c|}{ Pregnancy induced hypertensive disorder } \\
\hline Yes & $48(21.7)$ & $25(5.7)$ & $4.617(2.759-7.726)$ & $2.69(1.351-5.357)^{*}$ \\
\hline No & 173(78.3) & $416(94.3)$ & Ref & Ref \\
\hline \multicolumn{5}{|c|}{ Premature Rupture of membrane (PROM) } \\
\hline Yes & $28(12.7)$ & $30(6.8)$ & $1.988(1.155-3.420)$ & $1.669(0.850-3.278)$ \\
\hline No & 193(87.3) & $411(93.2)$ & Ref & Ref \\
\hline \multicolumn{5}{|l|}{ Onset of labor } \\
\hline Spontaneous & 184(83.3) & $412(93.4)$ & Ref & Ref \\
\hline Induced & $37(16.7)$ & $29(6.6)$ & $2.857(1.705-4.787)$ & $1.858(0.924-3.738)$ \\
\hline \multicolumn{5}{|l|}{ Prolonged second stage of labor } \\
\hline Yes & $35(15.8)$ & $27(6.1)$ & $2.885(1.697-4.907)$ & $2.630(1.399-4.944) *$ \\
\hline No & 186(84.2) & $414(93.9)$ & Ref & Ref \\
\hline \multicolumn{5}{|l|}{ Meconium stained liquor } \\
\hline Yes & $50(22.6)$ & $18(4.1)$ & $6.871(3.897-12.117)$ & $6.955(3.721-13.001) *$ \\
\hline No & 171(77.4) & 423(95.9) & Ref & Ref \\
\hline \multicolumn{5}{|l|}{ Mode of delivery } \\
\hline SVD & $99(44.8)$ & $313(71.0)$ & Ref & Ref \\
\hline Operative Vaginal & $39(17.6)$ & $47(10.7)$ & $2.623(1.622-4.244)$ & $1.568(0.868-2.835)$ \\
\hline $\mathrm{CS}$ & $69(31.2)$ & $73(16.6)$ & $2.988(2.005-4.455)$ & $2.005(1.223-3.287) *$ \\
\hline Vaginal Breech & $14(6.3)$ & $8(1.8)$ & $5.533(2.255-13.576)$ & $2.679(0.597-12.024$ \\
\hline \multicolumn{5}{|l|}{ Gestational Age } \\
\hline Term & 173(78.3) & 396(89.8) & Ref & Ref \\
\hline Late Preterm (34-36+6 weeks) & $41(18.6)$ & $29(6.6)$ & $3.236(1.947-5.378)$ & $1.354(0.666-2.750)$ \\
\hline \multicolumn{5}{|l|}{ Post term } \\
\hline & $7(3.2)$ & 16(3.6) & $1.001(0.405-2.478)$ & $0.981(0.333-2.894)$ \\
\hline
\end{tabular}




\begin{tabular}{|lcccc|} 
Vertex & $196(88.7)$ & $427(96.8)$ & Ref & Ref \\
Breech & $23(10.4)$ & $11(2.5)$ & $4.555(2.177-9.530)$ & $2.080(0.590-7.330)$ \\
Non-vertex & $2(0.9)$ & $3(0.7)$ & $1.452(0.241-8.762)$ & $2.019(0.313-13.036)$ \\
\hline Fetal birth weight & & & & \\
\hline 2500 - 3999 gram & $166(75.1)$ & $413(93.7)$ & Ref & Ref \\
$<2500$ gram & $52(23.5)$ & $24(5.4)$ & $5.391(3.217-9.032)$ & $4.380(2.216-8.657)^{*}$ \\
$\geq 4000$ gram & $3(1.4)$ & $4(0.9)$ & $1.866(0.413-8.428)$ & $2.655(0.474-14.876)$ \\
\hline
\end{tabular}

*: Significantly associated factors at a p-value $<0.05$

Ref: Reference group

\section{Supplementary Files}

This is a list of supplementary files associated with this preprint. Click to download.

- TableSuplmentarynew.docx 